\title{
Editorial: Announcing the operations management research best paper award
}

\author{
Scott Shafer • Jan Olhager
}

Published online: 14 December 2012

(C) Springer Science+Business Media New York 2012

In our last editorial entitled "the next leg for Operations Management Research," we ended by emphasizing that the most critical aspect for the continued success of $O M R$ is that researchers in our field continue to contribute their high quality research to the journal. In particular, research that utilizes, extends, and/or develops theory in order to advance practice.

In support of OMR's goal to publish high quality research that advances the practice of operations management through theory, we are pleased to announce the Operations Management Research Best Paper Award. In an effort to be consistent with the Wickham Skinner awards offered by POMS and the Jack Meredith Award offered by JOM, the OMR Best Paper Award will include plaques and cash prizes of $\$ 1,000$ for first place and $\$ 500$ for the runner up.

In addition to bringing $O M R$ to parity with other leading operations management journals, we believe that establishing the best paper award accomplishes two other important objectives. First, it provides another avenue to recognize and enhance the visibility of exceptional research in our field. Second, we hope that the opportunity for additional recognition creates an incentive for researchers to submit their best research to $O M R$ which in turn will help enhance the reputation of the journal. And of course, having another high quality outlet for our research benefits all researchers in the field.

\footnotetext{
S. Shafer $(\bowtie)$

Schools of Business, Wake Forest University,

Worrell Professional Center,

P.O. Box 7659, Winston-Salem, NC 27109, USA

e-mail: shafersm@wfu.edu

J. Olhager

Department of Industrial Management and Logistics, Lund University, Box 118, SE-22100 Lund, Sweden

e-mail: Jan.Olhager@tlog.lth.se
}

Consistent with the mission of the journal, papers will be evaluated on the extent to which they advance practice through theory. In addition, their overall presentation quality will also be considered.

More specifically, in terms of advancing practice, papers will be evaluated in terms of their relevance to practice, the extent to which important new insights are offered, the timeliness of the topic, and the ease with which the ideas and methodologies presented in the paper can be directly applied to practice. While advancing practice is necessary, it alone does not result in an exceptional contribution. Rather, truly exceptional papers advance practice by utilizing, extending, and/or developing theory. Therefore, papers will also be evaluated in terms of their rigor, the methodologies used, their innovativeness, and the extent to which the paper addresses an important gap in the literature.

Finally, the overall presentation quality of papers will also be evaluated. Along this dimension papers will be assessed in terms of the extent to which the writing is clear and concise, the paper has a clear and comprehensive abstract, is well organized with a logical flow, contains a clear discussion of the contribution to both practice and theory, discusses implications for future research, and is generally interesting.

All papers published in a volume including invited guest editorials are eligible to receive the best paper award. Each year, the Editor-In-Chief(s) will form a Best Paper Award Review Committee comprising current or past Associate Editors who collectively represent a variety of research areas and research methodologies. The Editor-In-Chief(s) will also nominate candidate papers for the award and have the members of the Best Paper Award Review Committee score and rank each of the nominated papers. The Editor-In-Chief (s) will select the paper to receive the Best Paper Award based on the collective rankings of the Best Paper Award Review Committee.

So, as we concluded our last editorial, keep submitting your good research to $O M R$ ! 